\title{
On the opacity change required to compensate for the revised solar composition (Research Note)
}

\author{
J. Christensen-Dalsgaard ${ }^{1}$, M. P. Di Mauro ${ }^{2}$, G. Houdek ${ }^{3}$, and F. Pijpers ${ }^{1}$ \\ 1 Department of Physics and Astronomy, Building 1520, Aarhus University, 8000 Aarhus C, Denmark \\ e-mail: [jcd; fpp] @phys . au.dk \\ 2 INAF - IASF, Istituto di Astrofisica Spaziale e Fisica Cosmica, via Fosso del Cavaliere 100, 00133 Roma, Italy \\ e-mail: mariapia.dimauro@iasf-roma.inaf.it \\ 3 Institute of Astronomy, Madingley Road, Cambridge CB3 0HA, UK \\ e-mail: hg@ast.cam.ac.uk
}

Received 9 May 2008 / Accepted 26 October 2008

\begin{abstract}
Context. Recent revisions of the determination of the solar composition have resulted in solar models in marked disagreement with helioseismic inferences.

Aims. The effect of the inferred composition change on the models is largely caused by the change in opacity. Thus, we wish to determine an intrinsic opacity change that would compensate for the revision to the composition.

Methods. By comparing models computed with the old and revised compositions we determine the required opacity change. Models are computed with the opacity modified in this manner and used as reference in helioseismic inversions to determine the difference between the solar and model sound speed.

Results. An opacity increase ranging between around 30 per cent close to the base of the convection zone and a few percent in the solar core leads to a sound-speed profile, for the revised composition, that is essentially indistinguishable from the original solar model. As a function of the logarithm of temperature this is well represented by a simple cubic fit. The physical realism of such a change remains debatable, however.
\end{abstract}

Key words. Sun: abundances - Sun: interior - Sun: helioseismology

\section{Introduction}

The opacity in the solar interior, and hence the solar internal structure, depends sensitively on the abundances of the heavy elements (e.g. Turck-Chièze et al. 2001a; Turck-Chièze \& Talon 2008). Recent analyses of the solar spectrum have led to substantial revisions of the solar abundances, particularly of oxygen, carbon, and nitrogen (for a review, see Asplund 2005). Relative to previous work, these studies had the advantage of being based on three-dimensional hydrodynamical models of the solar atmosphere and accounting for departures from local thermodynamic equilibrium. Also, unlike earlier analyses, they provided consistent abundance determinations from different spectral lines. As a result of the revision, the ratio $Z_{\mathrm{s}} / X_{\mathrm{s}}$ between the present solar surface abundances by mass of heavy elements and hydrogen was determined to be 0.0165 , corresponding, in calibrated solar models, to $Z_{\mathrm{s}}=0.0125$. For comparison, the commonly used composition of Grevesse \& Noels (1993) yields $Z_{\mathrm{s}} / X_{\mathrm{s}}=0.0245$, resulting in $Z_{\mathrm{s}}=0.0181$.

As pointed out, e.g., by Basu \& Antia (2004), Montalbán et al. (2004), Turck-Chièze et al. (2004), Bahcall et al. (2005), and Delahaye \& Pinsonneault (2006), this revision has substantial effects on solar models, greatly increasing the difference between their internal sound speed and the sound speed inferred from helioseismology. As an example, we consider Model S of Christensen-Dalsgaard et al. (1996), using the Grevesse \& Noels composition and OPAL opacities from Iglesias et al. (1992), and a corresponding model based on the new abundances and updated OPAL opacities (Iglesias \& Rogers 1996). Figure 1 shows sound-speed differences between the Sun and these two models, inferred through inversion of the "Best Set" of observed frequencies of Basu et al. (1997), combining data obtained with the BiSON network and the LOWL instrument (for further details on the inversion, see Christensen-Dalsgaard \& Di Mauro 2007). Large differences are also found in the depth of the convection zone and the envelope helium abundance between models based on the revised composition and the helioseismically inferred values. Chaplin et al. (2007) found that their analysis of low-degree solar oscillations strongly supported the old heavyelement abundance; on the other hand, the results of the analysis by Houdek \& Gough (2007), with careful inclusion of the influence of the outer layers of the Sun, indicated a heavy-element abundance somewhat lower than the Model S value, although substantially above the value obtained with the revised abundances. The effects of the new composition on solar models, as tested by helioseismology, were reviewed by Basu \& Antia (2008). Guzik (2006) provided an overview of the, largely unsuccessful, attempts to modify the assumptions in the model computation to compensate for the composition change.

By far the most important effect on solar modelling of the heavy-element abundance arises through the opacity. Thus, Montalbán et al. (2004) found that a substantial opacity increase close to the base of the convection zone would help in reducing 


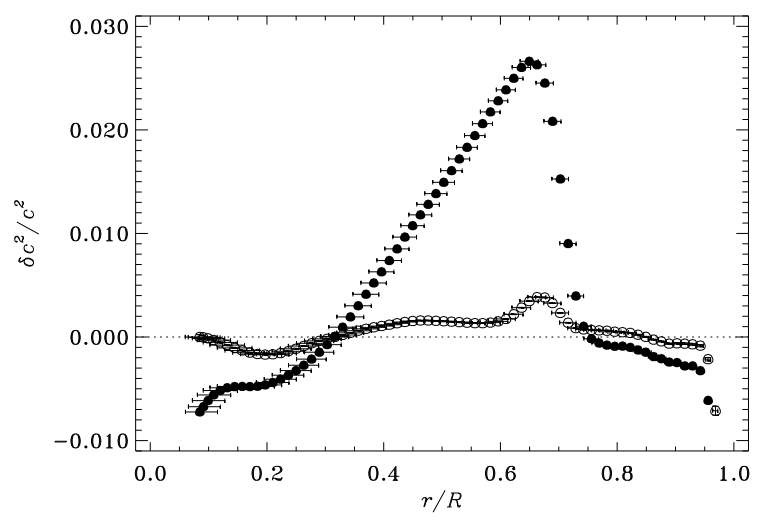

Fig. 1. Inferred relative differences in squared sound speed, in the sense (Sun) - (model), from inversion of the "Best Set" of observed frequencies of Basu et al. (1997). The open circles show results for Model S of Christensen-Dalsgaard et al. (1996), using the old solar composition, while the filled circles show results for a corresponding model, assuming the revised composition. The horizontal bars indicate the resolution of the inversion, while the vertical bars (hardly visible on this scale) show the $1-\sigma$ errors in the inferences.

the discrepancy caused by the revised abundances. Similarly, it was noted by Bahcall et al. (2005) that an intrinsic change in the opacity could be used to correct the model computation, and they estimated that an opacity increase of around 11 per cent over a relatively broad range in temperature would be required. Indeed, there are undoubtedly significant uncertainties in the complex opacity calculations. Following Bahcall et al. (2005), we estimate the intrinsic change in the opacity required to obtain a model structure corresponding closely to Model S, but with the revised composition.

\section{Determination of the opacity change}

The goal is to determine an opacity modification that would enable the sound-speed structure of Model S to be reproduced approximately with the revised solar surface composition. With just this constraint we can clearly only determine a modification that depends on a single variable, which we take to be temperature $T$. Thus, we write the modified opacity as

$$
\log \tilde{\kappa}(\rho, T, X, Z)=\log \kappa^{(\mathrm{Asp})}(\rho, T, X, Z)+f(\log T),
$$

where $\rho$ is density, $X$ and $Z$ are the abundances by mass of hydrogen and heavy elements, $\kappa^{(\text {Asp) }}$ is the opacity evaluated with the revised heavy-element composition, and log denotes logarithm to base 10 . The goal is to determine $f(\log T)$ such that $\tilde{\kappa}$ evaluated for a structure corresponding to Model S, but with the revised heavy-element composition, matches the opacity $\kappa^{(\mathrm{GN})}$ in Model S, evaluated for the original Grevesse \& Noels (1993) heavy-element composition.

To estimate $f(\log T)$, we consider two models of the present Sun: one is Model $\mathrm{S}$, characterized by $\left\{\rho_{\mathrm{S}}, T_{\mathrm{S}}, X_{\mathrm{S}}, Z_{\mathrm{S}}\right\}$, as functions of position in the model, and the second is Model Asp, similarly characterized by $\left\{\rho_{\text {Asp }}, T_{\text {Asp }}, X_{\text {Asp }}, Z_{\text {Asp }}\right\}$. We determine the difference in $\log \kappa$, at fixed $T$, between the two models as

$$
\begin{aligned}
\delta_{T} \log \kappa= & \log \kappa^{(\mathrm{GN})}\left(\rho_{\mathrm{S}}(T), T, X_{\mathrm{S}}(T), Z_{\mathrm{S}}(T)\right) \\
& -\log \kappa^{(\mathrm{Asp})}\left(\rho_{\mathrm{Asp}}(T), T, X_{\mathrm{Asp}}(T), Z_{\mathrm{Asp}}(T)\right),
\end{aligned}
$$

making explicit that the models are computed with $\kappa^{(\mathrm{GN})}$ and $\kappa^{(\text {Asp) }}$, respectively. Using the required property, Eq. (1), of $\tilde{\kappa}$ and

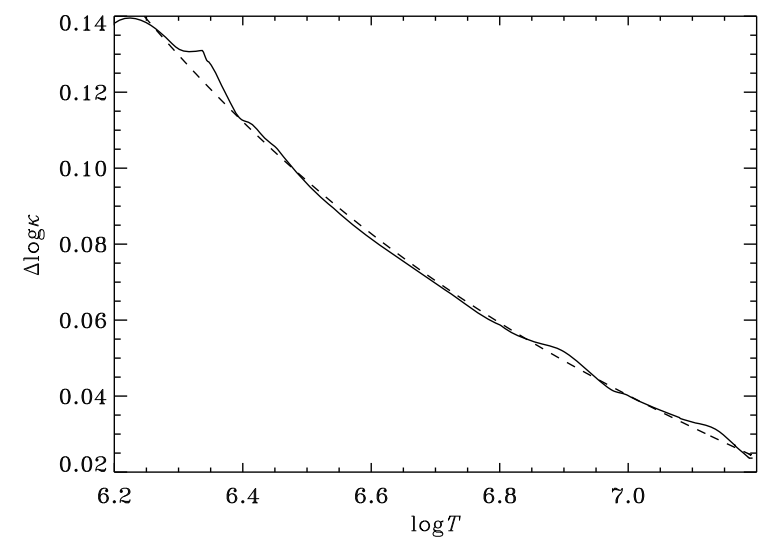

Fig. 2. The solid curve shows the intrinsic opacity change $\Delta \log \kappa=$ $f(\log T)$ determined from Eq. (4), on the basis of models with the old (Model S) and the revised (Model Asp) composition. The dashed curve shows the cubic fit $f_{\text {approx }}$ to $\Delta \log \kappa$, given by Eq. (5).

linearizing in model differences, we obtain

$$
\begin{aligned}
\delta_{T} \log \kappa \simeq & f(\log T)+\left(\frac{\partial \log \kappa}{\partial \log \rho}\right)_{T, X} \delta_{T} \log \rho \\
& +\left(\frac{\partial \log \kappa}{\partial \log X}\right)_{T, \rho} \delta_{T} \log X
\end{aligned}
$$

neglecting the effect of the different dependence of $Z$ on position in the two models; here, e.g., $\delta_{T} \log \rho=\log \rho_{\mathrm{S}}(T)-\log \rho_{\text {Asp }}(T)$. A similar expression was obtained by Bahcall et al. (2005). Hence, neglecting the relatively modest effects of the differences in $X$ and $Z$ at fixed $T$, we obtain the required opacity change as

$\Delta \log \kappa=f(\log T) \simeq \delta_{T} \log \kappa-\left(\frac{\partial \log \kappa}{\partial \log \rho}\right)_{T, X} \delta_{T} \log \rho$.

This procedure can be iterated to compensate for the error in the linearization and the neglect of the composition effects. In practice, we found that two iterations are sufficient to reach a model closely matching Model S.

\section{Results}

Figure 2 shows the resulting opacity change, mainly for the radiative interior, which is evidently the only region for which the change is relevant. We computed a full evolution sequence assuming the revised surface composition and applying the correction $\Delta \log \kappa$ to the opacity, and, as for Model S, calibrating the resulting Model $S^{\prime}$ to solar luminosity and radius as well as to the revised value of $Z_{\mathrm{s}} / X_{\mathrm{s}}$. Differences between Model $\mathrm{S}^{\prime}$ and Model $\mathrm{S}$ are illustrated in Fig. 3. It is evident that Model $\mathrm{S}^{\prime}$ closely matches Model S. To test how such a model change affects the comparison with the helioseismic inference, Fig. 4 shows the sound-speed inversion using this model as a reference. Clearly, it matches the helioseismic results as well as does Model S.

The simple dependence of $f(\log T)$ on $\log T$ makes it natural to approximate it by a low-order polynomial. In Fig. 2, the dashed line shows the following fit:

$f_{\text {approx }}(\log T)=0.1298-0.1856 \xi+0.1064 \xi^{2}-0.0345 \xi^{3}$,

with $\xi=\log T-6.3$, obtained through a least-squares fit with uniform weight to $f(\log T)$, for $\log T>6.3$. The result of using the approximation (5) rather than the original $f(\log T)$ in the 


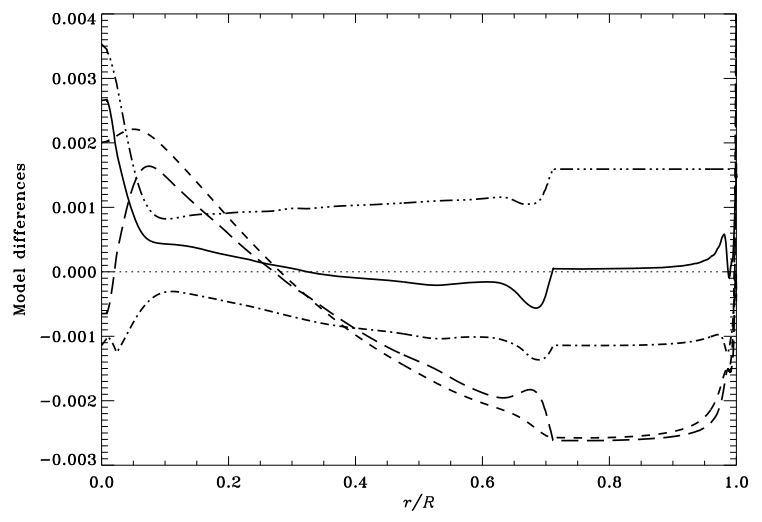

Fig. 3. Differences between Model $\mathrm{S}^{\prime}$, computed with the revised composition and the opacity change $\Delta \log \kappa$ illustrated in Fig. 2, and Model $\mathrm{S}$, in the sense (Model $\left.\mathrm{S}^{\prime}\right)-($ Model $\mathrm{S}): \delta \ln c^{2}$ (continuous), $\delta \ln p$ (short dashed), $\delta \ln \rho$ (long dashed), $\delta \ln T$ (dot-dashed) and $\delta X$ (triple-dot-dashed). Here ln is natural logarithm.

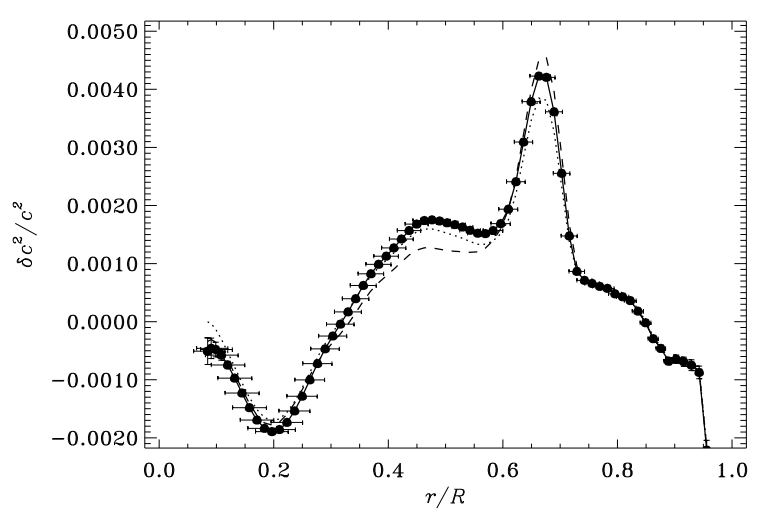

Fig. 4. Inferred relative differences in squared sound speed, in the sense (Sun) - (model), from inversion of the "Best Set" of Basu et al. (1997). The symbols with horizontal and vertical bars (see caption to Fig. 1) show results for Model $\mathrm{S}^{\prime}$, computed with the revised composition and applying the opacity correction $\Delta \log \kappa$ shown in Fig. 2. The dashed curve shows differences for Model $\mathrm{S}^{\prime \prime}$, similarly computed using the fit in Eq. (5). For comparison, the dotted curve shows the inferred difference for the original Model S.

evolution calculation, calibrating the model as before, and using the resulting Model $S^{\prime \prime}$ as a reference in a sound-speed inversion, is also shown in Fig. 4. This is barely distinguishable from the results of using the original $f(\log T)$.

In Table 1 we finally list the depth $D_{\text {cz }}$ of the convection zone and the envelope helium abundance $Y_{\mathrm{e}}$ for the models considered. For comparison, the helioseismically inferred value of $D_{\mathrm{cz}}$ is around 0.287 , and $Y_{\mathrm{e}}$ has been determined to be around 0.25 , although with some sensitivity to the equation of state used in the solar modelling (for a review, see Basu \& Antia 2008). It is evident that Model Asp is inconsistent with the observational measurements, whereas the remaining models are essentially in accordance with the observations.

\section{Discussion and conclusion}

The change in $\log \kappa$ presented in Fig. 2 corresponds to an opacity change of around 30 per cent close to the base of the solar convection zone, decreasing to a modest level in the core. This immediately raises the question of whether such a change is physically realistic. Comparisons between independent
Table 1. Properties of solar models (see text for a description). $D_{\mathrm{cz}}$ is the depth of the convective envelope, given in units of the solar radius $R_{\odot}$, and $Y_{\mathrm{e}}$ is the helium abundance in the envelope.

\begin{tabular}{lcc}
\hline \hline Model & $D_{\mathrm{cz}} / R_{\odot}$ & $Y_{\mathrm{e}}$ \\
\hline $\mathrm{Asp}$ & 0.2712 & 0.2286 \\
$\mathrm{~S}$ & 0.2885 & 0.2447 \\
$\mathrm{~S}^{\prime}$ & 0.2882 & 0.2489 \\
$\mathrm{~S}^{\prime \prime}$ & 0.2875 & 0.2488 \\
\hline
\end{tabular}

opacity calculations (e.g. Badnell et al. 2005) (see also Basu $\&$ Antia 2008, for a review) indicate that the uncertainty in the opacities in the relevant temperature range is less than 5 per cent, far lower than the required change. On the other hand, although highly sophisticated, the present opacity calculation might neglect effects that are significant. Kurucz (personal communication) noted that the neglect of a large number of elements of low abundance could have a significant effect on the Rosseland mean opacity, which is sensitive to even weak absorption in spectral bands unaffected by lines of the more abundant elements.

It is perhaps relevant to recall a similar situation when Simon (1982) made a plea for the re-examination of opacity calculations in the light of problems with modelling certain pulsating stars; he suggested that an increase in the opacity by a substantial factor could remove the discrepancies between models and observations. Although Magee et al. (1984) claimed that such an increase would be "incompatible with atomic physics", it was in fact supported by the OPAL opacity calculations (e.g. Iglesias \& Rogers 1991; Rogers \& Iglesias 1992), as a result of the inclusion of the effects of a large number of lines. Based on this experience, one should perhaps be cautious about excluding the possibility of substantial opacity modifications.

It is interesting that even the early helioseismic sound-speed inferences of Christensen-Dalsgaard et al. (1985) indicated a need for an increase in the then current opacity tables, as subsequently confirmed by the OPAL calculations. Tripathy et al. (1998) determined the opacity correction to the OPAL tables required to reproduce the difference between the helioseismically inferred sound speed and the sound speed in Model S, using the sensitivity of sound speed to opacity changes determined by Tripathy \& Christensen-Dalsgaard (1998); they found that a relative difference in opacity of approximately 5 per cent was required. Gough (2004) determined the opacity difference between Model S and a helioseismically calibrated solar model, finding a relative difference of approximately 1.5 per cent.

We have assumed that the opacity correction is a function of temperature alone. This is obviously a gross, if unavoidable, simplification that should be kept in mind if the correction obtained here is used in other stellar-model calculations. We also emphasize that the fit given in Eq. (5) is valid only in the range [6.3, 7.2] in $\log T$ over which it was obtained. Even so, it might be interesting to use an opacity change similar to the one obtained here in other stellar-model computations, such as the isochrone analysis of M 67 presented by VandenBerg et al. (2007), which indicated a preference for the old composition.

Given the difficulties that arise in solar modelling due to the revised solar abundances, it is obviously crucial to carry out further tests of the results. Furthermore, the properties of the computed atmosphere models should be tested against other relevant observations (e.g. Ayres et al. 2006). The complexity of the hydrodynamical modelling of the solar atmosphere makes independent calculations highly desirable, and it is encouraging that such calculations are now under way (Steffen 2007; Caffau et al. 2007). Caffau et al. (2008) made a determination of the 
solar oxygen abundance, resulting in a value intermediate between the old and new compositions considered here. We expect that the corresponding opacity correction required to reproduce Model S, obtained as in the present analysis, would be approximately half the value shown in Fig. 2. Interestingly, Holweger (2001) obtained an oxygen abundance consistent with the value found by Caffau et al.; Turck-Chièze et al. (2004) demonstrated that this implied a sound-speed difference relative to the helioseismic inferences that was in-between the results for the old and new compositions considered here.

Alternative independent determinations of the abundances of the relevant elements would obviously be valuable. An interesting possibility, proposed by Gong et al. (2001) and reviewed by Basu \& Antia (2008), would be to constrain the heavy-element abundance from helioseismic inference of its effect on the thermodynamic state, and hence the sound speed, in the solar convection zone. This is an extension of the successful helioseismic determination of the solar-envelope helium abundance (e.g. Vorontsov et al. 1991; Kosovichev et al. 1992; Antia \& Basu 1994), following the suggestion of Gough (1984) and Däppen \& Gough (1986). The results of these analyses remain uncertain: Lin \& Däppen (2005) found slight indications that the standard (Grevesse \& Noels 1993) heavy-element abundance was too high, whereas Antia \& Basu (2006) and Lin et al. (2007) concluded that the helioseismic results confirmed the original abundances. Further analysis and investigations of the effects of the uncertainties in the equation of state, as well as higherquality data including modes of higher degree, are required to obtain more definitive results.

The relatively limited goal of the present note is to investigate the compensating changes to the opacity required by the revision in the solar heavy-element composition, in order to reproduce models computed with the earlier abundance determinations. This illustrates one aspect of the sensitivity of the solar structure, as probed by helioseismology, to the physics of the solar interior. It is evident that a broader goal of helioseismology is to understand the full range of solar internal microphysics and dynamics required to obtain a model in accordance with the helioseismic inferences. As discussed above, this may involve further adjustments of the opacity; however, it is likely that other processes, such as weak mixing in the region below the convection zone, must be invoked (e.g. Brun et al. 1999; Christensen-Dalsgaard \& Di Mauro 2007). Through such suitable adjustments to the physical prescriptions used in the modelling, it has been possible to construct seismic solar models that reproduce the inferred sound speed (e.g. Turck-Chièze et al. 2001b); a more interesting question is evidently the physical basis for these adjustments.

Acknowledgements. We are grateful to D. O. Gough, R. L. Kurucz and A. Weiss for useful conversations. This work was supported by the European Helioand Asteroseismology Network (HELAS), a major international collaboration funded by the European Commission's Sixth Framework Programme.

\section{References}

Antia, H. M., \& Basu, S. 1994, ApJ, 426, 801

Antia, H. M., \& Basu, S. 2006, ApJ, 644, 1292

Asplund, M. 2005, ARA\&A, 43, 481

Ayres, T. R., Plymate, C., \& Keller, C. U. 2006, ApJS, 165, 618
Badnell, N. R., Bautista, M. A., Butler, K., et al. 2005, MNRAS, 360, 458

Bahcall, J. N., Basu, S., Pinsonneault, M., \& Serenelli, A. M. 2005, ApJ, 618, 1049

Basu, S., \& Antia, H. M. 2004, ApJ, 606, L85

Basu, S., \& Antia, H. M. 2008, Phys. Rep., 457, 217

Basu, S., Chaplin, W. J., Christensen-Dalsgaard, J., et al. 1997, MNRAS, 292, 243

Brun, A. S., Turck-Chièze, S., \& Zahn, J. P. 1999, ApJ, 525, 1032 (Erratum: ApJ, 536, 1005)

Caffau, E., Steffen, M., Sbordone, L., Ludwig, H.-G., \& Bonifacio, P. 2007, A\&A, 473, L9

Caffau, E., Ludwig, H.-G., Steffen, M., et al. 2008, A\&A, 488, 1031

Chaplin, W. J., Serenelli, A. M., Basu, S., et al. 2007, ApJ, 670, 872

Christensen-Dalsgaard, J., \& Di Mauro, M. P. 2007, in Stellar Evolution and Seismic Tools for Asteroseismology: Diffusive Processes in Stars and Seismic Analysis, ed. C. W. Straka, Y. Lebreton, \& M. J. P. F. G. Monteiro (Les Ulis, France: EDP Sciences), EAS Publ. Ser., 26, 3

Christensen-Dalsgaard, J., Duvall, T. L., Gough, D. O., Harvey, J. W., \& Rhodes Jr., E. J. 1985, Nature, 315, 378

Christensen-Dalsgaard, J., Däppen, W., Ajukov, S. V., et al. 1996, Science, 272, 1286

Däppen, W., \& Gough, D. O. 1986, in Seismology of the Sun and the distant Stars, ed. D. O. Gough (Dordrecht: Reidel), 275

Delahaye, F., \& Pinsonneault, M. 2006, ApJ, 649, 529

Gong, Z., Däppen, W., \& Nayfonov, A. 2001, ApJ, 563, 419

Gough, D. O. 1984, Mem. Soc. Astron. It., 55, 13

Gough, D. 2004, in Equation-of-State and Phase-Transition Issues in Models of Ordinary Astrophysical Matter, ed. V. Čelebonović, W. Däppen, \& D. Gough (Melville, New York: AIP), AIP Conf. Proc., 731, 119

Grevesse, N., \& Noels, A. 1993, in Origin and evolution of the Elements, ed. N. Prantzos, E. Vangioni-Flam, \& M. Cassé (Cambridge: Cambridge Univ. Press), 15

Guzik, J. A. 2006, in Proc. SOHO 18 / GONG 2006 / HELAS I Conf. Beyond the spherical Sun, ed. K. Fletcher (Noordwijk, The Netherlands: ESA Publications Division), ESA SP-624

Holweger, H. 2001, in Proc. Solar and Galactic Composition: A Joint SOHO/ACE Workshop, ed. R. F. Wimmer-Schweingruber, (Melville: American Institute of Physics), AIP Conf. Proc., 598, 23

Houdek, G., \& Gough, D. O. 2007, in Unsolved Problems in Stellar Physics, ed. R. J. Stancliffe, J. Dewi, G. Houdek, R. G. Martin, \& C. A. Tout (Melville: American Institute of Physics), AIP Conf. Proc., 948, 219

Iglesias, C. A., \& Rogers, F. J. 1991, ApJ, 371, L73

Iglesias, C. A., \& Rogers, F. J. 1996, ApJ, 464, 943

Iglesias, C. A., Rogers, F. J., \& Wilson, B. G. 1992, ApJ, 397, 717

Kosovichev, A. G., Christensen-Dalsgaard, J., Däppen, W., et al. 1992, MNRAS, 259,536

Lin, C.-H., \& Däppen, W. 2005, ApJ, 623, 556

Lin, C.-H., Antia, H. M., \& Basu, S. 2007, ApJ, 668, 603

Magee, N. H., Merts, A. L., \& Huebner, W. F. 1984, ApJ, 283, 264

Montalbán, J., Miglio, A., Noels, A., Grevesse, N., \& Di Mauro, M. P. 2004, in Proc. SOHO 14 - GONG 2004: Helio- and Asteroseismology: Towards a golden future, ed. D. Danesy (Noordwijk, The Netherlands: ESA Publication Division), ESA SP-559, 574

Rogers, F. J., \& Iglesias, C. A. 1992, ApJS, 79, 507

Simon, N. R. 1982, ApJ, 260, L87

Steffen, M. 2007, in Convection in Astrophysics, ed. F. Kupka, I. W. Roxburgh, \& K. L. Chan (Cambridge: International Astronomical Union and Cambridge University Press), Proc. IAU Symp., 239, 36

Tripathy, S. C., \& Christensen-Dalsgaard, J. 1998, A\&A, 337, 579

Tripathy, S. C., Basu, S., \& Christensen-Dalsgaard, J. 1998, in Poster Volume, Sounding Solar and Stellar Interiors, ed. J. Provost, \& F. X. Schmider, Université de Nice, Proc. IAU Symp., 181, 129

Turck-Chièze, S., \& Talon, S. 2008, Adv. Space Res., 41, 855

Turck-Chièze, S., Nghiem, P., Couvidat, S., \& Turcotte, S. 2001a, Sol. Phys., 200, 323

Turck-Chièze, S., Couvidat, S., Kosovichev, A. G., et al. 2001b, ApJ, 555, L69

Turck-Chièze, S., Couvidat, S., Piau, L., et al. 2004, Phys. Rev. Lett., 93, 211102

VandenBerg, D. A., Gustafsson, B., Edvardsson, B., Eriksson, K., \& Ferguson, J. 2007, ApJ, 666, L105

Vorontsov, S. V., Baturin, V. A., \& Pamyatnykh, A. A. 1991, Nature, 349, 49 